\title{
Adaptive Smoothing Method Based on Fuzzy Theory Study and Realization
}

\author{
Yao Wan 1 , Changlin $\mathrm{Hu}^{2}$ \\ ${ }^{1}$ The Second Department, Nanjing Institute of Electronic Technology, Nanjing, China \\ ${ }^{2}$ Computer Science, Nanjing South East University, Nanjing, China \\ Email: tuckhere@126.com
}

Received February 2015

\begin{abstract}
In this paper, we study about a method to optimize the fused track quality in intelligence network of radar target fusion system, considering the role of people in the fusion system; we start to find ways to optimize the quality of the fused track, and adaptive smoothing method is proposed based on fuzzy theory. Tests show that this method can greatly improve the quality of the fused track system for battlefield reconnaissance provides high-quality, high-reliability battlefield.
\end{abstract}

\section{Keywords}

\section{Secondary Smoothing, Fuzzy Reasoning, Adaptive Moving Average}

\section{Introduction}

Battlefield reconnaissance system handles all the information level by level, and the information includes radar reconnaissance, UAV reconnaissance, battlefield television reconnaissance, infrared reconnaissance, white reconnaissance, signal detection, human detection, and then fusing step by step, comprehensive analysis of the formation of a unified battlefield situation. Finally we assess the threat by the situation analysis assistant. In reconnaissance systems, through multi-sensor information fusion, acquisition integration of information can greatly improve the combat effectiveness of battlefield reconnaissance.

Multi-sensor information fusion system as an important part of battlefield reconnaissance system is highly valued and widely used in the military. One of its main tasks is, within the observational data from multiple sensors transferred to a treatment center or a master through integrated intelligence or data fusion, to establish clear goals of high-quality composite track report. In this system, due to a large amount of information to be used to detect, the reliability of the system is improved greatly. Because of the different detection properties of different sensors, such as different sensor's random error, and measurement system error, it comes to the poor quality of smoothness. This problem can be solved in two ways: first, before the integration of information quality assessment, the assessment results are used to guide the information fusion strategies to get high-quality fused track; second, a feedback mechanism for fusion track adaptive optimization is use. In this paper, the second approach, the adaptive fuzzy theory is used to optimize the parameters of the track after fusion adaptive optimization process, greatly improving the quality of the integration of information. 
Contents of this paper are organized as follows: first, the fuzzy theory and track smoothing methods outlined, followed by adaptive smoothing method which was based on fuzzy theory to design, and then simulation was used to validate the method; the final summary was given at last.

\section{Theoretical Basis}

Fuzzy theory [1] is based on the "expert knowledge", using an artificial intelligence rules, said the policy language, which is a complete set of basic theory and design, integrated approach. Vague language, fuzzy logic systems, fuzzy reasoning is an important part of fuzzy theory. The basic idea of fuzzy reasoning method is to use fuzzy sets to simulate human thinking and reasoning processes. It belongs to a class of uncertain reasoning. It is based on Zadeh fuzzy set theory was first proposed in 1965 [2].

Information integration is the utilization of multiple sources of information to extract higher quality than any single integrated information process information [3]. At present, the field has produced some uncertainty to the theory-based technology, which is represented by the Bayesian inference, evidence reasoning and fuzzy reasoning [4]. These technologies have their own characteristics, which can be carried out under unclear in relation reasoning connotation and extension of the concept of boundaries between concepts or broken case logic, it has a simple and effective prominent features, so has quite a number of areas has been successfully applied, such as artificial intelligence, fuzzy control, information integration, and military decision-making [5]-[7].

Track Smoothing [8] can be most directly improve the quality of information fusion, it attracted much attention of the user. Commonly used smoothing methods include: a causal analysis, simple average, moving average, trend extrapolation method. This smoothing method is nothing less than a comprehensive analysis and the reasons for and results from two angles of things, to find a smooth strategy or method. Causal analysis and trend extrapolation is based smoothing method analysis of the causes of things; simple average method and the moving average method is to analyze results from the development of things, to find ways to improve the smoothness.

\section{Adaptive Smoothing Method Based on Fuzzy Theory}

In the data fusion system, factors that affect the quality of the fused track are complex, these factors including performance indicators of different sensors, collaborative performance and environmental factors such as collaborative performance between multi-sensor, so we chose to depart from the results were fused track smoothing. Moving average method to improve, to get the second smoothing methods, and artificial intelligence (fuzzy inference) into its parameter adjustment, and to improve the quality of the fused track.

\subsection{Secondary Smoothing Method}

Right simple moving average smoothing method of heavy elements are equal, which is calculated as follows:

$$
S_{k-1}=\frac{Z_{k-1}+Z_{k-2}+\cdots+Z_{k-N}}{N}
$$

In this formulation, $S_{k-1}$ is the value of smooth at time $t ; N$ is the moving average of the number of sampling period; $Z_{k-1}$ is the measured value of the previous time; $Z_{k-2} Z_{k-3} Z_{k-N}$ denote $k-2$, i -3 until $k-N$ measurement time.

The value of smooth at time $k$ is:

$$
S_{k}=\frac{Z_{k}+Z_{k-1}+\cdots+Z_{k-N}}{N+1}
$$

By the Formula (1) and a moving average smoothing Equation (2) can be obtained in the form of recursive:

$$
S_{k}=\frac{N-1}{N} \cdot S_{k-1}+\frac{1}{N} \cdot Z_{k}
$$

Recursive form using Equation (3), twice the moving average, and seek a weighted average, you can get the second smoothing method. Formula of this method is simple, easy to implement. It retains the advantages of moving average method, but also reduce the amount of data storage; and because it is able to fully repair all his- 
torical data points evenly, to reflect changes in the trend data point is more accurate, and therefore get better smoothing effect. But the lack of a clear, choose the smoothing parameter-free method for determining the presence of which in the course of the actual workers can only work with a personal experience to choose. And the smoothing parameter is established, it cannot change the characteristics of time series based on the stage. Such smoothing model cannot truthfully, dynamically reflect the time sequence. In response to these problems, this paper designs a fuzzy adaptive smoothing model. The model implements adaptive dynamic smoothing of time series data with engineering practicality.

\subsection{Adaptive Smoothing Method Based on Fuzzy Theory}

The basic block diagram of the fuzzy adaptive smoothing system is shown in Figure 1. Error of the mean and variance for smooth real-time evaluation, based on the assessment results, the use of fuzzy reasoning method for real-time adjustment of the smoothing parameter, to reduce the error of the mean and variance smoothing purposes, thereby improving the overall quality of the integration of information.

\subsubsection{Select the Fuzzy Variable}

Analysis of multi-sensor data fusion system, the integration of track quality requirements: First, the fusion track as close to the real target track, which requires the integration of track with high accuracy; secondly the fusion track as smooth as possible, which requires integration track jitter of less high smoothness.

Therefore, the error of the mean $M_{x}$ and fused track error variance $V_{x}$ fuzzy rules as in the preceding paragraph, and the coefficient of variation smoothing parameter $C_{x}$ as a post-entry fuzzy rules.

First converting the input conventional fuzzy controller [9]. Figure 2 is the amount of blur for fuzzy logic for decision-making; a fuzzy relation $\mathrm{R}$ is determined in accordance with the decision-making control rules, fuzzy logic algorithm derived fuzzy output; final adjusted value clarity calculated to adjust the parameters to be adjusted.

\subsubsection{Blur}

The fusion track $M_{x}, V_{x}$ of the mean and variance of its fuzzy. The variable $M_{x}, V_{x}$ its expansion in one direction can be a variance of the variable domain, in this domain, in accordance with the mean distance between the variables describing the establishment of a collection of vague language, the use of triangular membership functions shown in Figure 3 the basic relationship figure.

Figure 3 are defined as fuzzy language: NL (negative big), NM (negative medium), NS (negative small), ZO (zero), PS (positive small), PM (middle), PL (CP). They represent a range domain, the probability of the center of each small domain of values for a description of the language, other languages describe the probability is zero

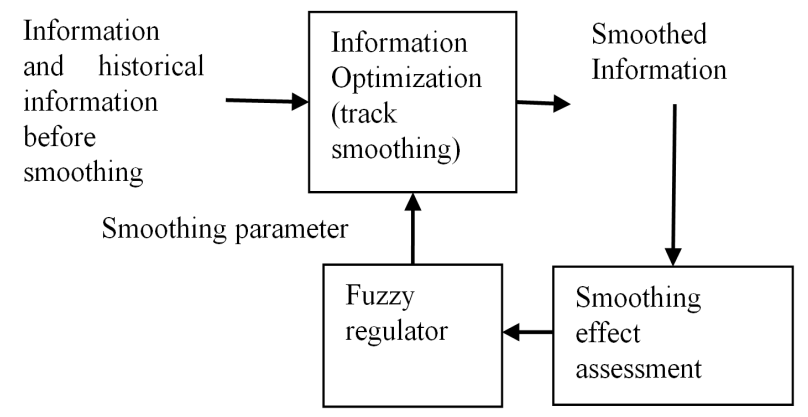

Figure 1. The basic block diagram of the fuzzy adaptive smoothing system.

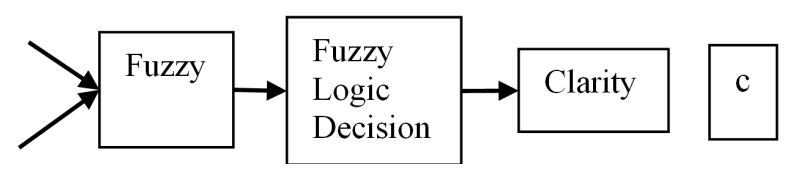

Figure 2. Conventional fuzzy regulator. 


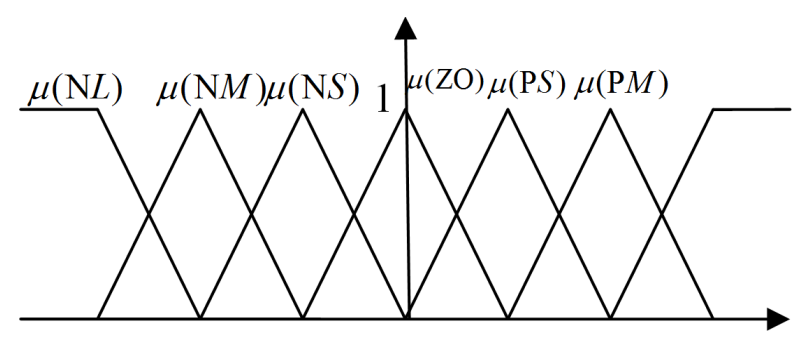

0

$\mu(\mathrm{P} L)$

Figure 3. Describes the fuzzy language map.

probability of corresponding points describe other triangle membership function MF, basically described as follows.

\subsubsection{Fuzzy Rules}

Obtained in accordance with the following basic rules of mathematical principles and engineering experience:

1) If the error is the mean $M_{x}$ is large, the smoothing parameter is increased;

2) If the error is the mean $M_{x}$ is small, reducing the smoothing parameters;

3) If the error variance $V_{x}$ is large, reducing the smoothing parameter;

4) If the error variance $V_{x}$ is small, increase the smoothing parameter.

Note: The size of $M_{x}$ is compared with reference values obtained by $\bar{M}_{x}$, The size of $V_{x}$ is compared with reference values obtained by $\bar{V}_{x}$.

According to the basic rules, designed to adjust the smoothing parameter of fuzzy rules, as described below, If $M_{x}$ is $A_{i}, V_{x}$ is $B_{i}$, then $C_{x}$ is $C_{i}$. Among them, $A_{i}$ is a fuzzy domain on the value of $M_{x}, B_{i}$ is a fuzzy domain on the value of $V_{x}, C_{i}$ is a fuzzy domain on the value of $C_{x}$. According to the fuzzy set of the language, which fuzzy rules shown in Table 1.

\subsubsection{Fuzzy Reasoning}

By fuzzy logic inference method [9] shows that for $n n$ fuzzy rules can be input-output relationship matrix $R_{1}$, $R_{2}, \ldots, R_{n}$, resulting in the total system of fuzzy relation matrix for the synthesis algorithm fuzzy rules

$$
R=\bigcup_{i=1}^{n} R_{i}
$$

Then for any $M_{x}$ and $V_{x}$, the corresponding fuzzy controller output $C_{x i j}$ is

$$
C_{x i j}=\left(M_{x i} \times V_{x j}\right) \circ R
$$

\subsubsection{Clarity}

Adjust the amount of blur $C_{x i j}$ on the Formula (5) was then precise calculations can be transferred to the direct regulation of the parameters. Here the weighted average method for fuzzy output clarity (i.e. defuzzification) treatment, (6) which is calculated as formula.

$$
c=\frac{\sum_{i=1}^{n} \mu_{C_{i}}\left(C_{i}\right) C_{i}}{\sum_{m=1}^{n} \mu_{C_{i}}\left(C_{i}\right)}
$$

\section{Tests}

The smoothing method previously designed for a fusion system, for real-time integration of the target track adaptive smoothing, 4 and 5 give the results of FIG. Figure 4 non-maneuvering target, which is fused track green before smoothing, yellow fused track smoothed. Figure $\mathbf{5}$ is a maneuvering target. Where green is fused 


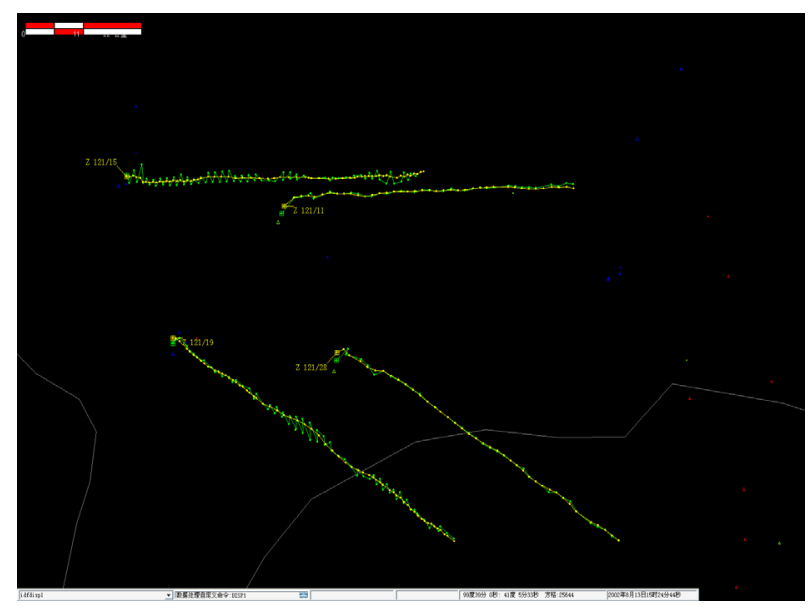

Figure 4. Fusion track (non-motorized target) track before and after smoothing.

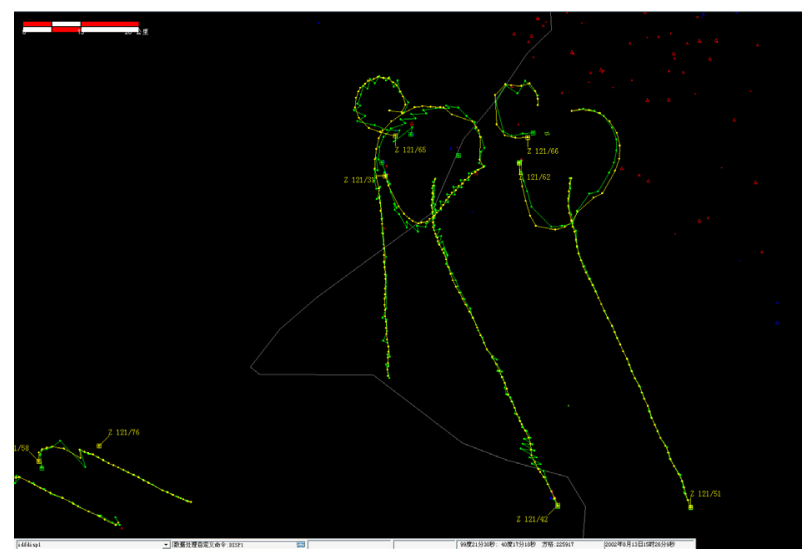

Figure 5. Fusion trajectory (maneuvering target) track before and after smoothing.

Table 1. Fuzzy rules.

\begin{tabular}{cccccccc}
\hline & NL & NM & NS & Z & PS & PM & PL \\
\hline NL & PL & PM & PS & Z & PS & PM & PL \\
NM & PL & PM & PS & Z & PS & PM & PL \\
NS & PM & PS & PS & Z & PS & PM & PL \\
Z & PM & PS & PS & Z & PS & PM & PL \\
PS & PM & PS & Z & NS & Z & PS & PM \\
PM & PS & Z & NS & NM & NS & Z & PS \\
PL & Z & NS & NM & NL & NM & NS & Z
\end{tabular}

track before smoothing, yellow fused track smoothed. Quality indicators were compared before and after the track and calculate the smoothed mean accuracy error which representative track, smoothness representative track of the error variance.

From Figure 4 and Figure 5, and Table 2 and Table 3 can be seen in both the non-maneuvering target is maneuvering target, the paper proposed smoothing method improves the precision and smoothness of the track, 
Table 2. Comparison of the mean and variance of the track error for each target before and after smoothing.

\begin{tabular}{ccccccc}
\hline \multirow{2}{*}{ Target NO. } & \multicolumn{3}{c}{ Mean of error $(\mathbf{k m})$} & \multicolumn{3}{c}{ Variance of error $(\mathbf{k m})$} \\
\cline { 2 - 7 } & Before & After & Rising rate (\%) & Before & After & Rising rate (\%) \\
11 & 0.6026 & 0.3888 & 35.48 & 0.2195 & 0.0983 & 55.23 \\
15 & 1.1863 & 0.5476 & 53.84 & 0.5956 & 0.1802 & 69.74 \\
19 & 1.2393 & 0.7019 & 43.37 & 0.3544 & 0.1936 & 45.39 \\
28 & 0.7124 & 0.4035 & 43.36 & 0.1862 & 0.0854 & 54.14 \\
\hline
\end{tabular}

Table 3. Comparison of the mean and variance of the front and rear track error for each target smooth.

\begin{tabular}{ccccccc}
\hline \multirow{2}{*}{ Target NO. } & \multicolumn{3}{c}{ Mean of error (km) } & \multicolumn{3}{c}{ Variance of error (km) } \\
\cline { 2 - 7 } & Before & After & Rising rate (\%) & Before & After & Rising rate (\%) \\
\hline 31 & 1.2486 & 0.7835 & 37.24 & 0.5792 & 0.2626 & 54.66 \\
42 & 1.0235 & 0.5769 & 43.63 & 0.5956 & 0.1802 & 69.74 \\
51 & 0.8699 & 0.5781 & 33.54 & 0.1014 & 0.0827 & 18.44 \\
62 & 0.9781 & 0.5564 & 43.11 & 0.4836 & 0.1977 & 59.12 \\
65 & 1.0538 & 0.6715 & 36.28 & 0.4472 & 0.1252 & 72.00 \\
66 & 1.5846 & 0.8244 & 47.97 & 0.4912 & 0.2010 & 59.07 \\
\hline
\end{tabular}

has greatly improved the quality of the fused track for battlefield reconnaissance battlefield provides high quality, high reliability.

\section{Summary}

The track filter smoothing method breaks through the concept of target modeling, and overcomes the high sensitivity of the sampling by track filter. Filtering divergence phenomenon caused by the physical environment and data model mismatch which has fundamentally overcame. Compared with other smoothing methods (such as fitting straight lines, moving mean, weighted mean, etc.), it takes full advantage of the historical information of target track and the trends of target location, eliminating the time-sensitivity of the algorithm. It greatly reduces the amount of storage (compared with the straight line fitting and the moving mean), and improves the computing speed. Meanwhile fuzzy theory is introduced to achieve the parameters of smooth which can be adjusted online intelligently, and it can better improve the precision of smooth.

\section{References}

[1] Zhu, J., et al. (1995) Fuzzy Control Theory and Applications. Machinery Industry Press, Beijing.

[2] Zadeh, L.A. (1965) Fuzzy Sets. Information Control, 8, 338-353. http://dx.doi.org/10.1016/S0019-9958(65)90241-X

[3] Waltz, E. and Llinas, J. (1990) Multisensor Data Fusion. Artech Housem, Norwood.

[4] Bu Kman, S.S. (1986) Multiple-Target Tracking with Radar Applications. Artech House, Norwood.

[5] Zhang, W.X. and Liang, Y. (1998) Principle of Uncertainty Reasoning. Xi'an Jiaotong University Press, Xi’an.

[6] Kleinla (1999) Sensor and Data Fusion Concepts and Applications. SPIE Optical Engineering Press.

[7] .Zhang, H.X. (1995) Ship-Based Fuzzy Inference Rules Avoidance Strategy. Fire Control and Command Control, 2.

[8] Billah, B., et al. (2006) Exponential Smoothing Model Selection for Fore-Casting. International Journal of Forecasting, 22, 239-247. http://dx.doi.org/10.1016/j.ijforecast.2005.08.002

[9] Wei, W. (2000) Intelligent Control Basis. Tsinghua University Press, Beijing. 